\title{
Convergência de recursos e mediação para inclusão digital: casos baianos
}

\section{Convergence of resources and mediation for digital inclusion: cases from Bahia}

\author{
Barbará Coelho Neves ${ }^{1}$ \\ Edvaldo Souza Couto ${ }^{2}$
}

\begin{abstract}
RESUMO
A partir dos estudos sobre a cibercultura, com ênfase na inclusão digital, o texto discute aspectos iniciais de uma pesquisa em desenvolvimento que procura aprofundar o tema da mediação e da convergência de recursos na inclusão digital no Estado da Bahia. Temos como objeto os pontos de inclusão digital (PID) de três municípios baianos verificados como potenciais para adoção do modelo de acesso baseado em letramento. A abordagem do estudo é qualitativa, descritiva e analítica. O trabalho defende e conclui que pensar a inclusão digital a partir da convergência dos recursos e da mediação pode proporcionar um salto de qualidade na realidade dos telecentros públicos baianos.
\end{abstract}

Palavras-chave: cibercultura; inclusão digital; mediação; telecentros; educação e comunicação.

\begin{abstract}
From studies of cyberculture, with emphasis on digital inclusion, this article discusses aspects of a research development that seeks to examine the subject of mediation and convergence of resources on digital inclusion in the State of Bahia. Our focus is on points of digital inclusion (PID) of three municipalities in Bahia checked as potential for the adoption of an access

${ }^{1}$ Mestrado em Ciência da Informação pela Universidade Federal da Bahia (UFBA). Professora do Curso de Extensão da Universidade Federal da Bahia, Brasil. E-mail: barbaracoelho2000@

${ }^{2}$ Doutorado em Educação pela Universidade Estadual de Campinas (UNICAMP). Professor Associado da Universidade Federal da Bahia, Brasil. E-mail: edvaldosouzacouto@gmail.com
\end{abstract} yahoo.com.br 
model based on literacy. The study approach is qualitative, descriptive and analytical. This paper argues and concludes that thinking about the digital inclusion from the convergence of resources view and from Mediation can provide a quality leap in the reality of public centers in Bahia.

Keywords: cyberculture; digital inclusion; mediation; telecentres; Education and communication.

\section{Introdução}

$\mathrm{O}$ acesso à informação pela sociedade civil tem sido apontado como um dos meios que pode contribuir para o desenvolvimento do potencial criativo e intelectual dos indivíduos. Esse processo de acesso, mediado por computador, vem sendo discutido na literatura de diversas áreas das Ciências Sociais Aplicadas e Humanas. Tais discussões vêm gerando estudos que, por sua vez, têm relatado e/ou embasado iniciativas governamentais e não governamentais que pretendem minimizar a exclusão digital no Brasil. Varias iniciativas de instituições - mantidas com recursos de origem privada, pública ou mista de mecanismos nacionais e internacionais - têm contribuído com a criação de espaços que proporcionam tipos de acessos variados à informação eletrônica, como os telecentros.

Nesse contexto, o trabalho apresenta e discute elementos iniciais de uma pesquisa em curso que procura observar e analisar aspectos da mediação em programas e projetos de inclusão digital no Estado da Bahia. Abordamos o contexto, o problema, a problemática, a relevância, a abordagem a que está circunscrita, o estado da arte do conhecimento e a justificativa de se desenvolver essa pesquisa em um curso de doutorado em Educação. $O$ trabalho está estruturado a partir de duas seções complementares. A primeira trata da contextualização, relevância e justificativa de se observar a inclusão digital pelo viés da formação dos sujeitos. A segunda apresenta os principais argumentos teóricos que fundamentam a pesquisa e delineia pontos de aproximações e convergência entre a teoria e a pesquisa que aborda tecnologia com mediação. Nas considerações finais, enfocamos o objeto de estudo, procurando relacionar as possibilidades de se considerar a convergência de recursos e aspectos da mediação para inclusão digital. Ao apresentar a proposta da pesquisa, o trabalho defende e conclui que pensar a inclusão digital a partir da convergência dos recursos e da mediação pode proporcionar um salto de qualidade na realidade dos telecentros públicos baianos. 


\section{Aspectos do contexto e justificativa do desenvolvimento da pesquisa}

O Programa Sociedade da Informação, exposto no conhecido "Livro Verde", propõe em um de seus objetivos "[...] contribuir para a efetiva participação social, sustentáculo da democracia política." (BRASIL, 2000, p. 6). Dentre os quesitos, destaca: importância da utilização da internet para o comércio eletrônico; divulgação de negócios e comunicação mais rápida e menos onerosa para as pequenas e médias empresas; geração de novas oportunidades de trabalho; e universalização do acesso (combatendo desigualdades e promovendo a cidadania) e a educação e aprendizado ao longo da vida (desenvolvendo competência). Esse cenário, idealizado pelo "Livro Verde", nos motivou a desenvolver esta pesquisa e a extrair o problema relacionado ao objeto ponto de inclusão digital (PID), na Bahia, baseando-se na polêmica entre os recursos da inclusão digital (Warschauer) e a mediação (Vygotsky).

No estágio inicial da pesquisa, o principal questionamento está relacionado à indagação sobre o que é a inclusão digital e o retorno à origem do termo, que tem revelado ainda muitas contradições diante dos significados e práticas nos diferentes contextos em que se apresenta. Nossa primeira questão é: quais elementos indicam que uma pessoa, município ou país é mais incluído digital que o outro? Essa indagação está inserida no entendimento de que não existe um ente de fato excluído da sociedade e que é preciso sucessivas aproximações do objeto para compreender sua realidade, sua essência. Se as tecnologias estruturam a sociedade, não há como de fato o sujeito ser excluído do social, nem das tecnologias que estruturam a vida cotidiana. Mas isto não quer dizer que os variados graus de inclusão fluam tranquilamente, nem que eles sejam dados igualitariamente aos sujeitos. Esse é um campo de tensões que envolve cultura tecnológica, políticas públicas, economia, educação para a inclusão social e digital.

No Brasil, a inclusão digital, muitas vezes, é um discurso político, do qual os governos se apropriam para desenvolver programas ou uma noção, ou seja, difundir o que a maioria das pessoas compreende e experimenta em relação ao acesso às tecnologias, sobretudo aos computadores e à internet. Desse modo, não há um conceito propriamente dito, mas uma série de elementos que apontam perspectivas que se baseiam em propostas voltadas para o acesso, treinamento ou formação de pessoas para a vida digital. Partindo dessa compreensão, localizamos este estudo na perspectiva que se faz da inclusão digital como um contexto da atual demanda de formação dos indivíduos frente às tecnologias de informação e comunicação (TIC) na sociedade contemporânea. 
Assim, este estudo sobre a convergência de recursos e mediação para inclusão digital revela-se importante para estreitar o tema e as relações da mediação com os aspectos da educação continuada, dos processos continuados de formação dos sujeitos, por localizar a questão no âmbito da educação e das políticas de educação. Também julgamos que o trabalho pode contribuir para a compreensão da temática no contexto social particular brasileiro, em especial na realidade da região Nordeste e, particularmente, na Bahia.

\section{Modelos e relevâncias de uma inclusão digital na perspectiva da mediação e convergência de recursos}

Atualmente, a discussão sobre a inclusão digital na perspectiva da mediação é muito criticada. Muitas vezes, as definições que abordam as questões são do ponto de vista estritamente técnico, por limitarem o problema ao acesso físico às ferramentas. Essa forte valorização do acesso físico faz com que, na maioria das vezes, as ações não considerem os conteúdos produzidos e difundidos. As condições do acesso físico às ferramentas são condições importantes para a inclusão e alfabetização digital, mas insuficientes para atender aos requisitos básicos, exigidos em um estágio mais avançado de inclusão, vislumbrado por autores que defendem a formação por meio do digital. Na sociedade da informação, os conteúdos e a cultura de produção e difusão são essenciais para a inserção participativa e plena dos sujeitos bem letrados digitalmente em processos como, por exemplo, a democracia eletrônica, inteligência coletiva e a cultura da colaboração.

Silveira (2011) defende que a condição do acesso físico à infraestrutura de conectividade pode proporcionar desigualdades de oportunidades no uso da rede. E chama atenção que não é suficiente conectar os cidadãos em redes precárias que não são compatíveis com a dinâmica que envolve a demanda e o trânsito da informação. Em 2005, Silveira esquematizou seis dimensões de acesso que o sujeito pode galgar na rede cibernética: a) o acesso à internet; b) o acesso aos conteúdos da internet; c) o acesso a e-mails; d) o acesso às linguagens básicas e instrumentos para usar a rede; e) o acesso às técnicas de produção de conteúdo; f) o acesso à construção de ferramentas e sistemas voltados às comunidades.

Percebemos, de acordo com Silveira (2005), que as atividades mais básicas, como ter acesso às máquinas e saber minimante usá-las, antecedem aquelas que envolvem o sujeito na produção de conteúdos e de sistemas mais complexos que produzem coletivamente e difundem conteúdos na rede e ampliam o processo de 
inclusão digital. O autor enfatiza que os níveis identificados são interdependentes, assim, é necessário ter acesso aos aparatos técnicos para que se possa alcançar o nível das atividades intelectuais em rede. O lugar de destaque do acesso às linguagens básicas e instrumentos, entre a técnica e o conteúdo, demonstra a relevância da mediação em apoio à formação intelectual.

Modelos como esses são importantes para esta pesquisa por apontarem que, além da demanda pela ampliação de infraestrutura, existem outros aspectos relevantes no processo de inclusão digital. Esses aspectos estão ligados diretamente à educação dos sujeitos. Nesses modelos, geralmente, os pontos que tratam de treinamento, capacidade intelectual e aprendizagem, criando significado para a vida, aparecem nos níveis mais superiores, posteriores à disponibilidade de infraestrutura e equipamentos. Nesta perspectiva, o acesso e o treinamento inicial para o uso dos equipamentos e das linguagens necessitam estar acompanhados de processos que envolvem aprendizagem em níveis que capacitam o sujeito a criar, a produzir conteúdo e potencializar, consequentemente, a geração de conhecimento.

Para sair do estado de acesso básico ou conectado aos equipamentos, é necessário um estágio intermediário que envolve aspectos que impactam na formação que leva à capacitação intelectual. Esse estágio intermediário imprescindível é o da mediação. A mediação aqui deve ser vista como uma estratégia de impulso, capaz de estimular o sujeito a avançar para chegar ao nível mais elaborado de promoção da inclusão digital, ou seja, aquele estado capaz de possibilitar aos usuários as condições para interagirem por meio da criação, da produção e da difusão de informações e conhecimentos.

Uma das causas diretas do acesso desigual à tecnologia digital na sociedade contemporânea está na distribuição do grande número de recursos. Esses recursos, segundo Dijk (2005, 2006), são os materiais (comumente associados à posse de equipamentos), mas também os temporais (considerando tempo de uso na nova mídia), os sociais (redes e nós que ajudam alcançar o acesso) e os culturais (o status e outras recompensas culturais que motivam pessoas a obterem acesso). A maneira como esses recursos estão distribuídos entre as pessoas pode explicar por que larga parcela da população está em posição de desigualdade social e digital. Estas desigualdades, como idade, etnia, sexo, inteligência, personalidade e saúde ou desability, aparecem relatadas nas diferentes oportunidades de acesso que as pessoas têm às novas mídias. A internet está no cerne do novo padrão sociotécnico e de organização. Esse processo global de desenvolvimento desigual talvez seja a expressão mais dramática da divisão digital.

Nosso estudo visa buscar e apresentar meios de fundamentar o elo entre inclusão digital e mediação para ampliar a capacitação intelectual em rede. Assim, nos interessam nesta perspectiva de inclusão digital que sejam considerados os aspectos da subjetividade da seguinte forma: 
a) considerar a experiência de um sujeito no processo de inclusão. A experiência que o sujeito sabe que incorpora e realiza em uma atividade que visa ao processamento da informação (FRAWLEY, 2000);

b) considerar que ao desenvolver uma atividade o sujeito deve ter consciência dela. A metaconsciência é também ação e instrumento (FRAWLEY, 2000). Este é um princípio básico na exploração de uma estratégia de busca de informação na web, por exemplo. A variedade de possibilidades (links e hiperlinks) favorece a dispersão dos objetivos. É necessária a consciência de ser consciente (VYGOTSKY, 1991) quando são desenvolvidas atividades no computador e na internet.

Segundo Morin (2009), o desenvolvimento da inteligência geral requer que seu exercício seja ligado a sua própria dúvida, como principal gatilho da atividade crítica. A cabeça bem feita que trata o entendimento do bom uso da inteligência geral, necessária para significação dos domínios da cultura científica e das humanidades, constitui uma interessante abordagem para tratar a tradução e reconstrução do conhecimento, principalmente em ambientes que podem proporcionar aprendizado com fontes de informação e usuários tão diversificados.

Observamos, nesse atual momento do movimento da inclusão digital, a conveniência de agregar recursos aos modelos de acesso (equipamentos, conectividade e letramento), com especial atenção ao recurso humano e digital. Nesses recursos residem as principais carências dos sujeitos na formulação do que buscar, onde buscar, como buscar, quando buscar, para que buscar e apresentação de ferramentas que mostram as maneiras pelas quais é possível reproduzir o resultado. A partir da relevância desses recursos é imprescindível o papel do mediador diante das fontes de informações disponíveis na internet, pois recuperá-las exige decisão no processo de planejamento da estratégia da recuperação. A mediação humana constitui um papel importante nesse processo. O mediador pode estimular a exploração de instrumentos intelectuais, desenvolvendo a crítica e a autonomia naqueles que utilizam e desenvolvem as aplicações da internet, potencializando o contexto de cultura digital.

Os três modelos de acesso às TIC, abordados por Warschauer (2003, 2006a), levam em consideração todas as particularidades de acesso às TIC por meio das iniciativas de inclusão digital, iniciando com dois modelos: "equipamentos" e "conectividade". Contudo, ele apresenta um terceiro, denominado de "letramento". Percebe-se que esse modelo constitui um ponto central de sua teoria, pois é a partir do modelo letramento que o autor desenvolve todo o seu argumento, provando a insuficiência dos modelos baseados no fornecimento de equipamentos e conectividade tão comuns nas iniciativas que visam desenvolver a inclusão digital. Então, seu raciocínio sobre o "modelo de acesso letramento" é relevante para o estudo que propomos, por nele constarem as categorias cha- 
madas de recursos físicos, digitais, humanos e sociais. Warschauer apresentou os recursos como essenciais em sua proposta de junção de tecnologias e inclusão social. Apropriando-se desses recursos, é possível chegar à seguinte proposta de um esquema de inclusão digital:

\section{Modelo de inclusão digital efetiva}
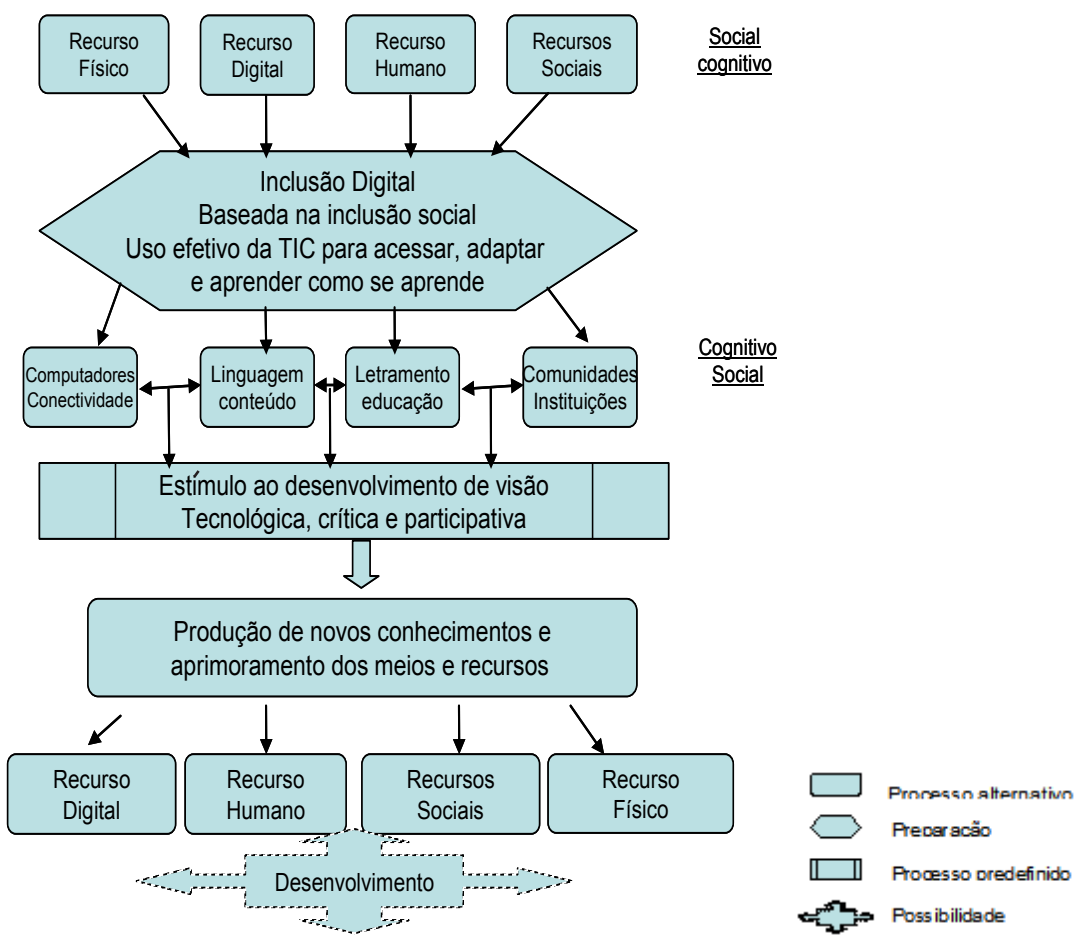

Esquema de inclusão digital baseada na convergência de recursos: importância tecnológica, crítica e participativa.

FONTE: NEVES (2010).

Os recursos físicos, computadores e conectividade, apresentam quem está conectado, o que pode ser feito e as iniciativas em vigência para conectar aqueles que ainda não estão. Segundo Warschauer (2006a, p. 79), "[...] o acesso físico equitativo em si não pressupõe capacidade equitativa de uso da TIC, que é influenciada por outros fatores [...]", como letramento, conteúdo e linguagem, educação e capital social. 
Os recursos digitais, conteúdo e linguagem, colocam em evidência a produção global e o seu acesso pelos diferentes tipos de usuários etc. Os “[...] governos, as organizações não governamentais e os grupos comunitários que procuram utilizar a internet em favor do desenvolvimento social têm de prestar muita atenção na questão de criação de conteúdo digital novo." (WARSCHAUER, 2006a, p. 121).

Os recursos humanos, letramento e educação, mostram associações entre tecnologia e letramento. Warschauer (2006a) vislumbra que os recursos humanos constituem o elo entre o recurso digital e o sujeito. A definição de letramento, no âmbito digital, está associada à habilidade de compreender os conteúdos disponíveis nas páginas da web. Com relação ao aspecto educacional deste recurso, o autor parte da perspectiva das comunidades de prática ou redes de pessoas dedicadas a atividades similares, aprendendo umas com as outras no processo. Neste ponto é que ele se aproxima do conceito de zona de desenvolvimento proximal (ZDP), elaborado por Vygotsky $(1991,2007)$ para explicar a importância da criação de redes informais em que tutores surgem naturalmente. Desse modo, no interior dos PID, a presença do monitor (mediador) pode cumprir um papel importante nesse processo de aprendizagem.

Os recursos sociais são as comunidades e instituições que abordam as relações sociais no ciberespaço, entendendo a internet como amplificadora do capital social das pessoas, assim como a importância do envolvimento da sociedade civil nas iniciativas de inclusão digital. É neste recurso que é observada a importância da participação ativa das comunidades, no desenvolvimento de projetos e preservação de sua infraestrutura. O estímulo e a valorização do capital social que a internet pode proporcionar à comunidade é um ponto interessante deste recurso.

Observando os recursos de Warschauer (2003, 2006a, 2006b), percebemos que todos eles são importantes para adaptar e criar conhecimento, estimulando, além da capacitação tecnológica, a formação com base no pensamento crítico e participativo do sujeito que poderá contribuir no processo de desenvolvimento (VYGOTSKY, 2008) por meio da produção de novos conhecimentos e aprimoramento dos meios e recursos. Pensar a inclusão digital acreditando que cada recurso é importante quando são adotados em convergência no PID está de acordo com a teoria de Warschauer e permite uma aproximação com as teses de Vygotsky (2007, 2008), pois computadores e internet sem linguagem e conteúdos adequados às necessidades individualizadas dos usuários não possuem muita importância enquanto facilitadores da "inclusão digital" em uma perspectiva que colabora com a formação.

Para Vygotsky (1991, 2007), a ideia de mediação é o mesmo que intermediação, ou seja, onde uma coisa se encontra interposta entre um ponto e outro com o intuito de relação. A ideia básica versa que a relação do homem com o mundo não é direta, mas mediada, seja por signos ou instrumentos. Por instrumentos compreen- 
de-se as ferramentas utilizadas pelo sujeito nas relações com o ambiente. Essas tecnologias são usadas para mediação da ação concreta do sujeito com o mundo.

Gomes (2006, p. 276) observou tal processo de mediação com relação à biblioteca, considerando-a como um espaço de mediação semiótica “[...] por atuar na mediação entre as ações de condensação, de expressão e de documentação dos conhecimentos produzidos e aquelas atividades realizadas pelos sujeitos leitores para revisitar e ressignificar os conteúdos acessados". Esse posicionamento corrobora a perspectiva do PID ser um espaço que possa provocar a construção de significados pelos sujeitos que ali acessam os conteúdos informacionais. Isso implica que, para que esses PID sejam considerados como "ambiente cultural e de aprendizagem", a mediação da informação é imprescindível.

O contato entre monitor "ativo" e os usuários - tanto no PID como de maneira remota -, sob a perspectiva de Vygotsky (2007), proporcionado pelo viés da interação, constitui uma oportunidade de criar possibilidade de internalização. A interação proporcionada por meio da orientação, por exemplo, é uma operação da atividade mediada que se desenvolve do externo para o interno. Uma situação como essa pode ser ilustrada, por exemplo, quando o monitor interage com um usuário, orientando-o a encontrar maneiras de resolver o seu problema relacionado à pesquisa escolar. Nessa ocasião, o monitor (mediador) permite que este crie uma espécie de espiral do desenvolvimento. Nessa perspectiva, ao invés de desenvolver a atividade de outrem, o monitor ajuda-o na atividade, quantas vezes forem necessárias, permitindo que o usuário avance todas as vezes que seu apoio for solicitado.

A figura a seguir mostra que o avanço, o desenvolvimento do sujeito, de um nível a outro não se dá de modo sequenciado, mas em acordo com o modelo espiral, onde as dificuldades são sempre retomadas, reelaboradas e mediadas, em busca de novas e criativas soluções.

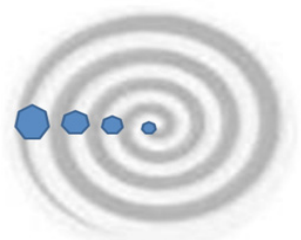

Desenvolvimento do indivíduo para os níveis superiores. Baseado em Vygotsky (2007).

Chamamos brevemente neste texto de monitor ativo aquele sujeito mais experiente, capaz de conduzir os usuários nas suas demandas no computador e internet. Este movimento do monitor na realidade de um PID pode ser estabelecido tanto com seu contato direto com os usuários quanto na condução de 
que o usuário possa se desenvolver a partir do entendimento de que é possível resolver suas demandas por meio de outros usuários, do tutorial disponível nos programas e do apoio remoto, ou seja, por meio de outros usuários on-line. Nessa abordagem do desenvolvimento em espiral de Vygotsky (2007), os usuários dos PID, ao passarem pelo mesmo ponto em uma atividade psicológica, mediada pelo monitor, podem avançar para um nível superior, sendo conduzidos à internalização da atividade, o que pode ocorrer desde o acesso aos conteúdos à seleção de uma ferramenta de busca de informações apropriada para sua demanda.

Com base neste fundamento, acreditamos que é inexorável a contribuição da confluência entre Warschauer (2005, 2006a) e Vygotsky (1995) para o tema da inclusão digital nos PID baianos que estudamos.

\section{Considerações finais}

Observamos 17 PID distribuídos em 10 municípios no Estado da Bahia, confirmando a hipótese que os modelos de acesso que estão sendo utilizados nos PID em municípios baianos, mapeados pela Fundação Getúlio Vargas (FGV) como os mais incluídos digitalmente, não vêm adotando uma abordagem cognitiva baseada na convergência de recursos. Entretanto, três desses PID pesquisados apresentaram potencial para adoção do modelo de acesso baseado em letramento, de acordo com o quadro a seguir:

SITUAÇÃO DOS PID QUANTO AO MODELO DE ACESSO

\begin{tabular}{|l|l|l|l|}
\hline \multirow{2}{*}{$\begin{array}{l}\text { MUNICÍPIO } \\
\text { PESQUISADO }\end{array}$} & \multicolumn{2}{|l|}{ PID SELECIONADO } & MODELO DE ACESSO \\
\cline { 2 - 4 } & Programa & Nome & Situação no período da pesquisa \\
\hline Salvador & PISD-BA & $\begin{array}{l}\text { CDC Biblioteca Pública } \\
\text { Monteiro Lobato }\end{array}$ & Potencial para o letramento \\
\hline Lauro de Freitas & PISD-BA & CDC Terreiro da Goméia & Potencial para o letramento \\
\hline Itabuna & $\begin{array}{l}\text { Estação } \\
\text { Digital }\end{array}$ & $\begin{array}{l}\text { Estação Digital de } \\
\text { Itabuna }\end{array}$ & Equipamentos e conectividade \\
\hline Feira de Santana & PISD-BA & CDC Maestro Miro & Equipamentos e conectividade \\
\hline Alagoinhas & MC & $\begin{array}{l}\text { Biblioteca Municipal de } \\
\text { Alagoinhas }\end{array}$ & Equipamentos e conectividade \\
\hline Mucuri & PISD-BA & $\begin{array}{l}\text { CDC Prefeitura de } \\
\text { Mucuri }\end{array}$ & Equipamentos e conectividade \\
\hline Santo A. Jesus & MC & $\begin{array}{l}\text { Rede de Desenvolvimen- } \\
\text { to Social (OSCIP) }\end{array}$ & Potencial para o letramento \\
\hline
\end{tabular}

NOTA: Embora a pesquisa tenha sido executada em 10 cidades, três delas não aparecem no quadro porque não apresentaram nenhum ponto de inclusão digital público em funcionamento naquele momento. 
De acordo com o quadro, os PID pesquisados em Salvador, Lauro de Freitas e Santo Antônio de Jesus apresentam potencial para implementação de um processo de inclusão digital na perspectiva da formação, balizado no modelo de acesso que Warschauer (2003) denominou de letramento e em aspectos da mediação.

Com isso, objetivamos na pesquisa, em fase de desenvolvimento, analisar por meio de uma pesquisa qualitativa, de tipo descritiva e analítica, os aspectos que indicaram o potencial desses três municípios para o desenvolvimento de uma inclusão digital na perspectiva da mediação e convergência de recursos. Para se aproximar com maior intensidade da realidade do contexto de inclusão digital destes municípios, elegemos 1 (um) PID em cada uma destas cidades com as características que indicaram potencialidade de mediação para inclusão digital no estudo que deu origem à problematização atual. Ressaltamos que os municípios de Lauro de Freitas e Santo Antônio de Jesus foram indicados no Mapa da Exclusão Digital da FGV como os menos incluídos entre os "10 mais" baianos.

Diante do exposto, para estruturarmos a pesquisa, temos a hipótese que os PID que possuem potencial para adoção do modelo de acesso baseado em letramento podem implementar uma nova perspectiva para inclusão digital baseada na convergência de recursos e na mediação.

Assim, compreendemos que para implementar uma abordagem que considera a mediação nos PID é imprescindível que se analise o conceito de zona de desenvolvimento próximo (ZDP). Trazer para uma pesquisa sobre inclusão digital tal conceito se constitui enriquecedor por dois motivos: primeiro, por apontar a necessidade de mediação humana nesses PID, pois, para serem reconhecidos como centros de informação, estes devem estar voltados ao desenvolvimento do usuário; segundo, por se perceber que a implementação dos PID pautada somente em disponibilização de infraestrutura, sem considerar aportes da formação, pode constituir um equívoco na perspectiva de sanar o gap entre incluídos e excluídos no novo contexto social e digital.

Entendemos a relevância de se considerar o mediador e os aspectos que estimulam o desenvolvimento pautado na junção dos recursos que envolvem os modelos de acesso técnico (computadores e conectividade) e o modelo de acesso baseado em letramento. Ao imaginar a possibilidade de que a inclusão digital pode acontecer sem o modelo baseado em letramento, pode-se estar igualando os PID às lan houses, que têm como foco principal a comercialização da oferta de acesso à internet e a equipamentos.

Este estudo tem por objetivo direto o desenvolvimento de uma tese de doutorado em Educação que aborda a inclusão digital na perspectiva da convergência de recursos e da mediação, utilizando como modelo, ou objeto de estudo em experiência, a investigação dos três PID baianos identificados como 
potenciais para adoção do modelo de acesso baseado em letramento. Ressaltamos que um salto qualitativo já foi verificado com este estudo, uma vez que os três PID classificados se estruturam a partir da apropriação dos recursos físicos, digitais, humanos e sociais, por meio de um entendimento que era necessário convergir-lhos nas iniciativas de inclusão digital. Além do desenvolvimento de tal convergência, também foi possível fazer aproximações com aspectos da mediação, por meio dos conceitos de zona de desenvolvimento próximo e mediação da informação. O que a pesquisa quer fazer é verificar e aprofundar os modos como a mediação é feita nesses PID e defender que essa experiência da mediação qualificada, sempre visando a estágios mais avançados de letramento digital, pode proporcionar um salto de qualidade na realidade dos telecentros públicos e, por conseguinte, na inclusão digital de baianos.

\section{REFERÊNCIAS}

BRASIL. Sociedade da informação: ciência e tecnologia para a construção da sociedade da informação no Brasil. Brasília, 2000.

DIJK, J.A.G.M. van. The Deepening divide: inequality in the information society. Thousand Oaks: Sage Publicaions, 2005. cation, 2006.

. The Network society: social aspects of new media. 2 ed. London: Sage Publi-

FRAWLEY, W. Vygotsky e a ciência cognitiva: linguagem e integração das mentes social e computacional. Porto Alegre: Artes Médicas Sul, 2000.

GOMES, H. F. Práticas pedagógicas e espaços informacionais da universidade: possibilidades de integração na construção do espaço crítico. 372f. Il. Tese (Doutorado em Educação) - Faculdade de Educação, UFBA, Salvador - Bahia, Brasil, 2006.

INSTITUTO de Brasileiro de Informação em Ciência e Tecnologia. Mapa da Inclusão Digital. Brasília, DF, 2007. (Não publicado).

MORIN, E. A cabeça bem feita. Rio de Janeiro: Bertrand Brasil, 2009.

QUIVY, R.; CAMPENHOUDT, L. V. Manual de investigação em ciências sociais: trajectos. Lisboa: Grandiva, 2003.

SILVEIRA, S. A. Exclusão digital: a miséria na era da informação. São Paulo: Fundação Perseu Abramo, 2005. 
. Para além da inclusão digital: poder comunicacional e novas assimetrias. In: BONILLA, M. H. S.; PRETTO, N. L. (Orgs.). Inclusão digital: polêmica contemporânea. Salvador, 2011.

VYGOTSKY, Lev S. Aprendizagem e desenvolvimento intelectual. In: LEONTIEV, A. et al. Psicologia e pedagogia: bases psicológicas da aprendizagem e do desenvolvimento. São Paulo: Moraes, 1991.

- A formação social da mente: o desenvolvimento dos processos psicológicos superiores. São Paulo: Martins Fontes, 2007.

. Pensamento e linguagem. Tradução de Jéferson L. Camargo. 4. ed. São Paulo: Martins Fontes, 2008.

WARSCHAUER, M. Technology and social inclusion: rethinking the digital divide: MIT, 2003. Disponível em: <http://www.mitpress.mit.edu/catalog/item/default. asp?sid=BAC81CFA-2B4A-44FF.html>. Acesso em: 03/07/2006.

. Going one-to-one. Association for Supervision and Curriculum Development, Educational Leadership, London, v. 62, n. 1, 2005.

. Tecnologia e inclusão social: a exclusão digital em debate. São Paulo: Senac, $2006 \mathrm{a}$.

. Laptops and literacy: learning in the wireless classroom. New York: Teaches College Press, 2006b.

. Learning the digital age. Educational Leadership, London, v. 63, n. 4, p. 34-38, dec. 2005-jan. 2006c.

Texto recebido em 21 de maio de 2011.

Texto aprovado em 24 de agosto de 2012. 